\title{
Análisis multivariado de los cambios hemáticos y morfométricos de Caiman latirostris y Caiman yacare según especie, sexo y estación del año
}

\author{
Barboza, N.N.; Panseri, A.F.; Mussart, N.B.; Koza, G.A.; Coppo, J.A. \\ Cátedra de Fisiología, Facultad de Ciencias Veterinarias, UNNE, Sargento Cabral 2139, Corrientes (3400), \\ Argentina. Tel.03783-425753. E-mail: fisiologia@vet.unne.edu.ar.
}

\begin{abstract}
Resumen
Barboza, N.N.; Panseri, A.; Mussart, N.B.; Koza, G.A.; Coppo, J.A.: Análisis multivariado de los cambios hemáticos y morfométricos de Caiman latirostris y Caiman yacare según especie, sexo y estación del año. Rev. vet. 21: 2, 112-122, 2010. Para optimizar la cría en cautiverio de los caimanes es necesario encontrar dietas que aceleren su crecimiento. Las dietas pueden evaluarse a través de los aumentos del peso, dimensiones corporales e indicadores nutricionales sanguíneos. El objetivo del ensayo fue obtener valores de referencia y variaciones fisiológicas de dichos parámetros en ejemplares juveniles de Caiman latirostris y Caiman yacare. En un criadero del nordeste argentino se dispuso de 207 caimanes $(50 \%$ de cada especie y sexo), que se alimentaron ad libitum con harina de carne y pellets balanceados (47 y $37 \%$ de proteínas respectivamente). En cada estación, durante un año se efectuaron pesajes, medición de 5 dimensiones corporales y determinación de 39 analitos sanguíneos. Los resultados, procesados mediante análisis multivariado de la variancia (MANOVA), no evidenciaron diferencias significativas entre sexos pero sí entre especies y entre estaciones $(\mathrm{p}<0,05)$. En promedio, albúmina, glucosa, calcio, magnesio, potasio, hemoglobina, HCM, CHCM, VCM, GGT, longitud total, longitud hocico-cloaca, ancho de cabeza, perímetro torácico y peso fueron más altos en C. latirostris. En cambio, proteínas totales, globulinas, ácido úrico, colesterol total, triglicéridos, C-LDL, sodio, cobre, hematocrito, eritrocitos, leucocitos, CPK, ALP, AST, CHE, LDH y longitud de cabeza fueron más altos en C. yacare. Los indicadores nutricionales revelaron valores más altos en verano, fenómeno que se atribuye al cese de la alimentación durante el letargo invernal de los caimanes. La ausencia de variaciones intersexuales debería interpretarse teniendo en cuenta que se trató de animales jóvenes, que aún no manifestaban comportamiento reproductivo. En conclusión, se obtienen valores de referencia para ejemplares de categoría sub-adultos de C. latirostris y C. yacare en cautiverio. El peso, las dimensiones morfométricas y los valores hemáticos variaron según la especie y época del año. Se espera que estos conocimientos puedan ser aplicados para mejorar el sistema de cría de caimanes autóctonos.
\end{abstract}

Palabras clave: Caiman latirostris, Caiman yacare, peso, dimensiones, valores sanguíneos, variaciones fisiológicas.

\begin{abstract}
Barboza, N.N.; Panseri, A.; Mussart, N.B.; Koza, G.A.; Coppo, J.A.: Hematological and morphometrical multivariate analysis of changes due to species, sex, and season of the year in Caiman latirostris and Caiman yacare. Rev. vet. 21: 2, 112-122, 2010. To optimize breeding systems of captive caymans it is necessary to find diets able to accelerate their growth. The increases of weight, corporal dimensions and blood nutritional indicators are useful to evaluate the efficiency of a diet. The objective of this assay was to obtain reference values and physiological variations of these parameters in Caiman latirostris and Caiman yacare juvenile specimens. In a hatchery from Northeast Argentine, 207 caymans $(50 \%$ each species and sex), fed ad libitum with meat flour and balanced pellets (47 and 37\% of protein respectively), were studied. Weighing and determination of five corporal dimensions and thirty nine blood analytes were carried out in each season, during one year. Results were processed by means of multivariate analysis of the variance (MANOVA). Significant differences between species and seasons were found $(p<0.05)$, but not between sexes. On average, albumin, glucose, calcium, magnesium, potassium, hemoglobin, $\mathrm{MCH}, \mathrm{MCHC}, \mathrm{MCV}, \mathrm{GGT}$, total longitude, muzzle-tail longitude, head wide, thoracic perimeter and liveweight were higher in C. latirostris. On the other hand, total proteins, globulins, uric acid, total cholesterol, triglycerides, LDL-C, sodium, copper, hematocrit, erythrocytes, leukocytes, CPK,
\end{abstract}




\begin{abstract}
ALP, AST, CHE, LDH and head longitude were higher in C. yacare. Nutritional indicators revealed higher values in summer, circumstance that may be attributed to the cease of feeding during the caiman winter lethargy. The absence of intersexual variations should be interpreted considering that studied animals were young specimens with no manifestation of reproductive behavior. In conclusion, reference values for sub-adult category specimens of captive C. latirostris and C. yacare, are reported. Liveweight, morphometrical sizes, and biochemical values varied according to species and season of the year. Data gathered in this assay may be used to improve breeding systems of autochthonous caimans).
\end{abstract}

Key words: Caiman latirostris, Caiman yacare, liveweight, corporal dimensions, blood values, physiological variations.

\section{INTRODUCCIÓN}

En el nordeste argentino habitan dos especies autóctonas de crocodylidos de la familia Alligatoridae (Caiman latirostris y Caiman yacare) ${ }^{5}$. Con el fin de comercializar cuero y carne de estos reptiles, en los últimos años han proliferado en la zona criaderos donde se practica el sistema ranching, consistente en recolección de huevos del medio natural, con eclosión y ulterior crianza en condiciones controladas hasta alcanzar el tamaño comercial para su sacrificio y venta. Un aspecto importante de tal explotación es la devolución a su ambiente del porcentaje estimado de animales que hubieran sobrevivido en condiciones naturales ${ }^{16,27}$.

En diversos lugares del mundo, la investigación científica está abocada al objetivo de acelerar la velocidad de crecimiento de los caimanes en cautiverio, para tornar más rentable la producción. Ello implica encontrar dietas adecuadas en cantidad de alimento, calidad de sus componentes y digestibilidad de los principios nutritivos ${ }^{15}$. El uso de indicadores nutricionales sanguíneos, aunados a la evolución del peso y dimensiones corporales, puede coadyuvar al logro de dicho objetivo. La obtención del intervalo de referencia para valores de laboratorio también asume importancia para optimizar el diagnóstico de las enfermedades de los reptiles en cautiverio ${ }^{5,26}$.

El objetivo del ensayo fue obtener valores de referencia para C. latirostris y C. yacare de la misma edad (categoría sub-adultos), sometidos a la misma dieta, en dos estaciones: fría (otoño e invierno) y cálida (primavera y verano), así como verificar diferencias atribuibles al sexo.

\section{MATERIAL Y MÉTODOS}

Animales, instalaciones, alimentación. Se utilizaron en total 207 ejemplares de Caiman sp. clínicamente sanos (104 C. latirostris y 103 C. yacare), aproximadamente $50 \%$ de cada sexo (90 machos y 117 hembras). Se trató de animales "subadultos", con edades de 1-5 años, pesos de 2-7 kg y longitudes de $80-130 \mathrm{~cm}$. Al inicio del ensayo los caimanes fueron sexados de visu con la ayuda de un espéculo, identificándolos mediante caravanas.
Los reptiles estuvieron alojados en el criadero "El Cachapé" (Provincia de Chaco), en piletas techadas cuyo piso estaba cubierto con agua de napa en un $40 \%$, la cual se renovaba día por medio. En invierno dispusieron de calefacción (estufas a gas y paneles solares).

Los caimanes fueron alimentados ad libitum tres veces por semana, con partes iguales de harina de carne (materia seca 92,98\%; cenizas 24,42\%; proteína bruta $47,17 \%$; extracto etéreo $13,41 \%$; fibra bruta $2,42 \%$; extractivo no nitrogenado $3,04 \%$; fósforo $4,41 \%$; calcio $5,05 \%$ ) y pellets balanceados (materia seca $92,95 \%$; cenizas $8,79 \%$; proteína bruta $37,52 \%$; extracto etéreo $4,52 \%$; fibra bruta $4,73 \%$; extractivo no nitrogenado $44,34 \%$; fósforo $1,16 \%$; calcio $0,8 \%$ ).

Controles, toma de muestras. Los controles periódicos se efectuaron en cuatro oportunidades, en cada una de las estaciones del año, las cuales luego fueron agrupadas en estación cálida (primavera-verano) y estación fría (otoño-invierno).

Los pesajes se efectuaron en una balanza colgante y las mediciones de las dimensiones corporales con una cinta métrica metálica, a saber: longitud total (LT: desde el hocico al extremo distal de la cola), longitud hocico-cloaca (LHC: desde el hocico al borde anterior de la cloaca), longitud cabeza (LC: desde el hocico al cóndilo occipital), ancho cabeza (AC: entre los cóndilos maxilares) y perímetro torácico (PT: a nivel de las axilas).

La sangre se extrajo con jeringa y aguja a partir del seno venoso post-occipital. Una alícuota fue tratada con anticoagulante (EDTA) y la otra fue centrifugada para obtener suero. Estas muestras se preservaron refrigeradas $\left(5^{\circ} \mathrm{C}\right)$ hasta su procesamiento en el laboratorio.

Determinaciones de laboratorio. Las pruebas hematológicas y bioquímicas se concibieron de tal manera que su espectro abarcara tanto la exploración del estado nutricional del animal como el de diversas funciones orgánicas factibles de ser alteradas por quebrantos de la salud ${ }^{3}$. Desde el punto de vista instrumental, la espectrofotometría se realizó en un aparato L.Mannheim 4010 UV-visible. Las determinaciones bioquímicas se efectuaron bajo control de calidad intralaboratorial, utilizando patrones comerciales de comparación (Standatrol). La fotometría de llama se llevó a cabo en un 
aparato Metrolab 305-D con calibradores Biopur. Las electroforesis fueron realizadas en una cuba accionada por fuente de poder Chemar CHF-I-3 de amperaje regulable y los ferogramas fueron cuantificados en un densitómetro Citocon CT-440 digital, automático, provisto de impresora.

Eritrograma: el hematocrito se evaluó por centrifugación de tubos capilares a $12.000 \mathrm{rpm}$. La concentración de glóbulos rojos se obtuvo mediante recuento microscópico en hemocitómetro de Neubauer, empleando métodos y reactivos específicos para reptiles ${ }^{2,7}$. La determinación de hemoglobina se realizó por espectrofotometría, técnica de la cianmetaHb, lecturas a $540 \mathrm{~nm}$, reactivos Wiener Lab. Previamente fueron separados por centrifugación los núcleos libres de los eritrocitos hemolizados, como se recomienda ${ }^{2}$. A través de cálculos convencionales se estimaron los índices hematimétricos: volumen corpuscular medio (VCM), hemoglobina corpuscular media (HCM) y concentración de hemoglobina corpuscular media (CHCM).

Leucograma: los leucocitos totales se evaluaron por recuento a partir de frotis coloreados (método indirecto acorde a técnica aviar, colorantes Biopur) y la fórmula leucocitaria (heterófilos, linfocitos, monocitos, eosinófilos y basófilos) se determinó por recuento diferencial (200 células) a partir de frotis coloreados según May Grünwald-Giemsa, reactivos Biopur.

Proteinograma: las proteínas totales se valoraron por espectrofotometría (método del biuret, $540 \mathrm{~nm}$, reactivos Wiener). Las fracciones seroproteicas (albúminas y globulinas alfa, beta y gamma) se separaron por electroforesis en soporte de acetato de celulosa, buffer de veronal sódico y coloración amidoschwartz, con ulterior transparentización y cuantificación por densitometría. La relación albúmina/globulinas-RAG se obtuvo por cálculo.

Nitrógeno no proteico: por espectrofotometría fueron determinadas las concentraciones séricas de urea (técnica de la ureasa, $570 \mathrm{~nm}$, reactivos Wiener), creatinina (método del picrato alcalino, $510 \mathrm{~nm}$, reactivos Wiener) y ácido úrico (técnica enzimática de la uricasa, $505 \mathrm{~nm}$, reactivos Wiener).

Glucosa: técnica de la oxidasa/peroxidasa, lectura espectrofotométrica a $505 \mathrm{~nm}$, reactivos Wiener.

Lipidograma: incluyó las determinaciones de triglicéridos (técnica del glicerolfosfato-oxidasa/peroxidasa, $505 \mathrm{~nm}$, reactivos Wiener), colesterol total (colesterol-oxidasa-peroxidasa, $505 \mathrm{~nm}$, reactivos Wiener), colesterol ligado a lipoproteínas de alta densidad (C-HDL) y de baja densidad (C-LDL): precipitación selectiva de la lipoproteína y valoración enzimática del colesterol, reactivos Wiener, y lipoproteínas alfa y beta (separación por electroforesis en gel de agarosa, buffer de veronal y coloración Fat Red 7B Biopur, valoración por densitometría).

Ionograma: magnesio (método del xylidyl blue, lecturas a $510 \mathrm{~nm}$, reactivos Wiener), calcio (cresolftaleíncomplexona, $570 \mathrm{~nm}$, reactivos Wiener), fósforo inorgánico (técnica del molibdato-ascorbato, $620 \mathrm{~nm}$,
Tabla 1. Contribución del peso y las variables morfométricas y bioquímicas a los tres primeros componentes principales.

\begin{tabular}{|c|c|c|c|}
\hline Variable & $\mathrm{CP} 1$ & $\mathrm{CP} 2$ & $\mathrm{CP} 3$ \\
\hline Peso & $-0,01$ & 0,35 & 0,15 \\
\hline LT & $-0,13$ & 0,20 & 0,25 \\
\hline LHC & 0,04 & 0,20 & 0,18 \\
\hline $\mathrm{LC}$ & $-0,05$ & $-0,22$ & 0,19 \\
\hline $\mathrm{AC}$ & 0,09 & 0,31 & 0,13 \\
\hline PT & 0,04 & 0,34 & 0,07 \\
\hline Proteínas totales & $-0,05$ & $-0,17$ & 0,35 \\
\hline RAG & 0,15 & 0,14 & $-0,29$ \\
\hline Glucosa & 0,23 & $-0,08$ & 0,21 \\
\hline Colesterol total & $3,4 \times 10^{-3}$ & $-0,25$ & 0,21 \\
\hline Creatinina & 0,25 & $-0,11$ & $-0,03$ \\
\hline Ácido úrico & $-0,23$ & $-0,02$ & $-0,17$ \\
\hline Sodio & 0,12 & 0,12 & 0,10 \\
\hline Potasio & 0,25 & $-1,3 \times 10^{-3}$ & 0,14 \\
\hline Calcio & 0,26 & $-0,04$ & $-0,06$ \\
\hline Fósforo inorgánico & 0,22 & $-0,19$ & $-0,07$ \\
\hline Magnesio & 0,14 & 0,20 & $-0,12$ \\
\hline Cobre & $-0,16$ & 0,07 & $-0,30$ \\
\hline ALP & $-0,23$ & 0,16 & 0,09 \\
\hline ALT & 0,10 & 0,13 & $-0,17$ \\
\hline AST & $-0,25$ & $-0,15$ & $-0,01$ \\
\hline CPK & $-0,24$ & $-0,16$ & $-0,01$ \\
\hline Hematocrito & 0,11 & $-0,28$ & 0,10 \\
\hline VCM & 0,14 & 0,18 & 0,25 \\
\hline $\mathrm{HCM}$ & 0,25 & 0,11 & 0,02 \\
\hline $\mathrm{CHCM}$ & 0,24 & 0,03 & $-0,11$ \\
\hline Leucocitos & 0,11 & $-0,18$ & $-0,28$ \\
\hline Linfocitos & 0,23 & $-0,12$ & 0,11 \\
\hline Eosinófilos & 0,10 & $-0,02$ & $-0,32$ \\
\hline Heterófilos & $-0,23$ & 0,04 & $-0,03$ \\
\hline Monocitos & $-0,21$ & 0,19 & $-0,11$ \\
\hline Basófilos & 0,20 & 0,09 & $-0,15$ \\
\hline Eigenvalores & 13,15 & 6,50 & 6,00 \\
\hline Varianza acumulada (\%) & 41 & 61 & 80 \\
\hline
\end{tabular}

CP: componente principal; LT: longitud total; LHC: longitud hocico-cloaca; LC: longitud de la cabeza; AC: ancho de la cabeza; PT: perímetro torácico; RAG: relación albúminas / globulinas; ALP: fosfatasa alcalina; ALT: alanin aminotrasferasa; AST: aspartato aminotransferasa; CPK: creatin fosfoquinasa; VCM: volumen corpuscular medio; HCM: hemoglobina corpuscular media; CHCM: concentración de hemoglobina corpuscular media.

reactivos Wiener), cobre (método de la batocuproína, $480 \mathrm{~nm}$, reactivos Boehringer), sodio y potasio (fotometría de llama con calibradores y testigos Biopur).

Enzimograma: fosfatasa alcalina ALP (técnica del fenilfosfato aminoantipirina, lecturas a $520 \mathrm{~nm}, 37^{\circ} \mathrm{C}$, reactivos Wiener), gammaglutamil transpeptidasa GGT (glutamil nitroanilida, $405 \mathrm{~nm}, 30^{\circ} \mathrm{C}$, reactivos Wiener), creatinfosfokinasa CPK (ATPcisteína, 620 $\mathrm{nm}, 37^{\circ} \mathrm{C}$, reactivos Wiener), lactato dehidrogenasa LDH (NADlactato, $505 \mathrm{~nm}, 37^{\circ} \mathrm{C}$, reactivos Wiener), aspartato aminotransferasa AST, ex-GOT (aspartato- 
cetoglutarato, $505 \mathrm{~nm}, 37^{\circ} \mathrm{C}$, reactivos Wiener), alanin aminotransferasa ALT, ex-GPT (alaninacetoglutarato, $505 \mathrm{~nm}, 37^{\circ} \mathrm{C}$, reactivos Wiener) y butirilcolinesterasa CHE (cinética con butiriltiocolina, $405 \mathrm{~nm}, 30^{\circ} \mathrm{C}$, reactivos Wiener).

Procesamiento estadístico de los datos. La normalidad de los datos fue verificada mediante la prueba de Shapiro-Wilk. Las variables se clasificaron en diez grupos: peso, morfometría, proteinograma, nitrógeno no proteico, glucosa, lipidograma, ionograma, enzimograma, eritrograma y leucograma. Se realizó un análisis de correlación de Pearson para reducir el número de variables al momento de realizar el análisis multivariado.

Se confeccionó una matriz de datos que incluyó 32 variables bioquímicas y morfométricas obtenidas en 207 individuos. Con dicha matriz se realizó un análisis de componentes principales (CP) a fin de obtener el ordenamiento de las especies, sexos y estaciones del año (usado como criterio de clasificación), así como identificar las variables que contribuyeron a esa ordenación. A partir de las variables más significativas obtenidas del análisis de $\mathrm{CP}$ se realizó un análisis multivariado de la varianza (MANOVA de dos factores) y el test a posteriori de Bonferroni al 5\% para evaluar el grado de diferenciación entre las especies y los sexos de los sujetos experimentales. La variabilidad total no debida a las diferencias entre los grupos (variabilidad dentro de los grupos) fue evaluada mediante la prueba de Wilks' Lambda.
Los soportes informáticos usados para los análisis estadísticos fueron: Statistica (StatSoft Inc. 2001, Versión 6) e InfoStat (Grupo InfoStat FCA-Córdoba, Argentina, versión 2008).

\section{RESULTADOS Y DISCUSIÓN}

Componentes principales. El análisis de CP demostró que los tres primeros componentes principales (CP) expresaron un $80 \%$ de la varianza total. El CP 1 constituyó el $41,1 \%$ de la varianza y estuvo representado principalmente por creatinina, potasio, calcio, HCM y CHCM, de manera positiva, mientras que AST y CPK, lo hicieron de manera negativa. El CP 2 aportó el $20,3 \%$ adicional y estuvo representado positivamente por el peso y los parámetros morfométricos $\mathrm{AC}$ y PT, así como negativamente por el colesterol total y el hematocrito. El CP 3 asumió el 18,7\% de la variación total: las proteínas totales contribuyeron de manera positiva y el cobre, RAG, leucocitos totales y eosinófilos influyeron de manera negativa sobre este componente (Tabla 1).

El gráfico bidimensional que representa el ordenamiento de las especies, sexos y estaciones del año obtenido en el análisis de CP (Figura 1) reveló la existencia de al menos cuatro grupos distinguibles que se correspondieron a las dos especies de caimanes en las dos estaciones del año analizadas (cálida y fría). Comparando entre las especies, C. latirostris presentó valores bioquímicos y morfológicos más elevados que C. yacare. Dentro de cada una de las especies se pudo

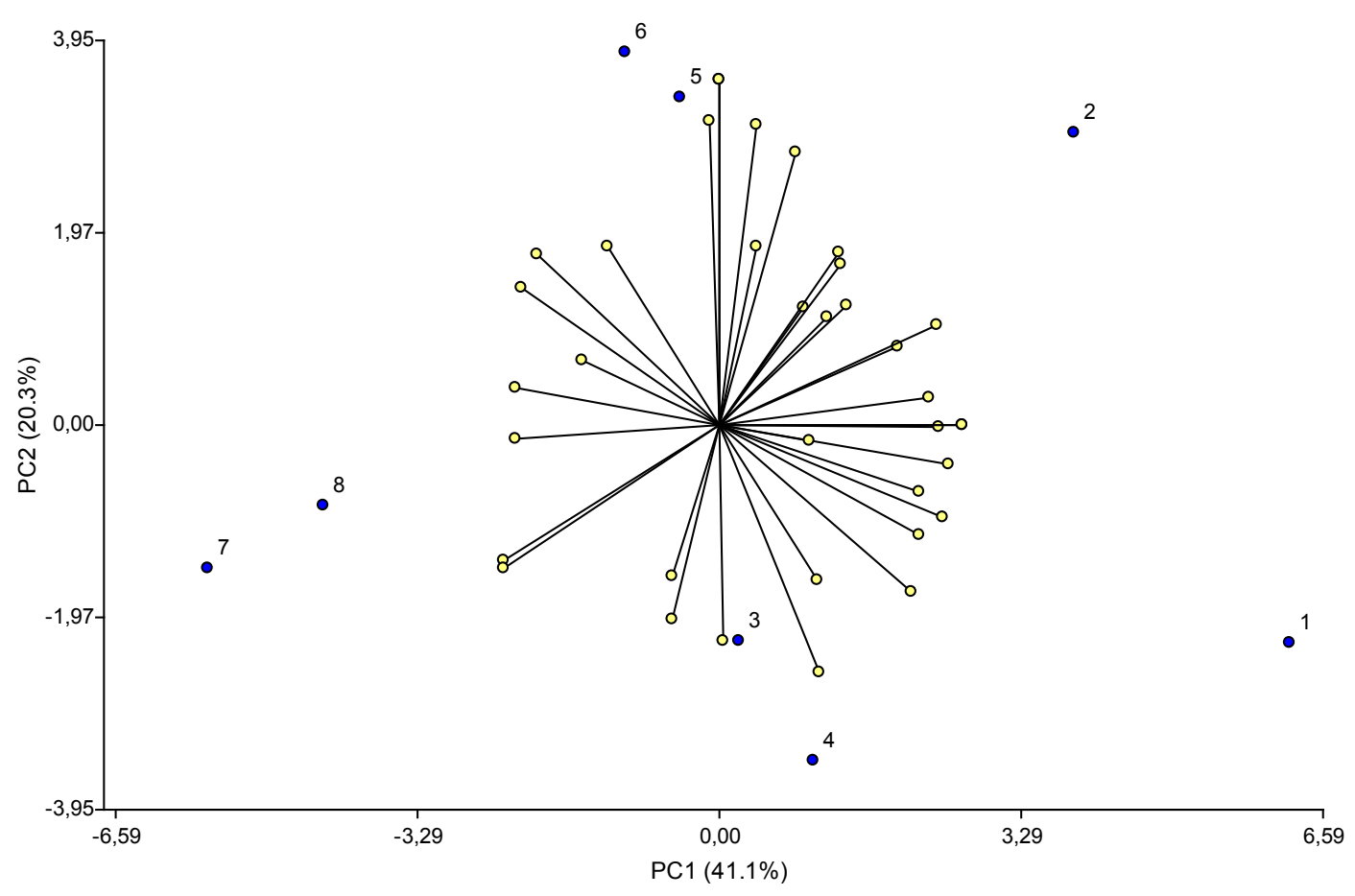

Figura 1. ACP de los parámetros peso, morfometría y valores bioquímicos de los caimanes. PC: componente principal; 1: warm station, C. latirostris male; 2: warm station, C. latirostris female: 3: warm station, C. yacare male; 4: warm station, C. yacare female; 5: cold station, C. latirostris male; 6: cold station, C. latirostris female; 7: cold station, C. yacare male; 8: cold station, C. yacare female. 
Tabla 2. Análisis de la variancia para los efectos especie, sexo e interacción.

\begin{tabular}{lcccccc}
\hline \multirow{2}{*}{ Variables } & \multicolumn{2}{c}{ Especie } & \multicolumn{2}{c}{ Sexo } & \multicolumn{2}{c}{ Especie*Sexo } \\
\cline { 2 - 7 } & $\mathrm{F}$ & $\mathrm{p}$ & $\mathrm{F}$ & $\mathrm{p}$ & $\mathrm{F}$ & $\mathrm{p}$ \\
\hline Peso & 5,367 & 0,022 & 0,687 & 0,408 & 3,108 & 0,080 \\
Proteínas totales & 6,866 & 0,010 & 2,496 & 0,116 & 1,411 & 0,237 \\
RAG & 13,132 & 0,000 & 3,296 & 0,071 & 1,818 & 0,179 \\
Glucosa & 0,230 & 0,632 & 3,943 & 0,049 & 3,270 & 0,072 \\
Colesterol total & 15,768 & 0,000 & 1,127 & 0,290 & 0,178 & 0,673 \\
Creatinina & 1,374 & 0,243 & 0,287 & 0,593 & 0,193 & 0,661 \\
Ácido úrico & 10,119 & 0,002 & 0,451 & 0,503 & 0,000 & 0,994 \\
Potasio & 1,542 & 0,216 & 1,638 & 0,202 & 1,872 & 0,173 \\
Calcio & 0,778 & 0,379 & 0,161 & 0,688 & 0,054 & 0,816 \\
AST & 20,543 & 0,000 & 0,013 & 0,908 & 0,000 & 0,994 \\
CPK & 11,932 & 0,001 & 0,593 & 0,442 & 0,077 & 0,782 \\
VCM & 1,868 & 0,173 & 1,815 & 0,180 & 0,599 & 0,440 \\
HCM & 9,905 & 0,002 & 4,594 & 0,033 & 0,626 & 0,430 \\
CHCM & 5,717 & 0,018 & 1,656 & 0,200 & 0,012 & 0,913 \\
Leucocitos & 0,066 & 0,798 & 0,037 & 0,848 & 0,267 & 0,606 \\
\hline Wilks' Lambda & 6,108 & 0,000 & 1,248 & 0,241 & 0,720 & 0,762 \\
\hline
\end{tabular}

Las abreviaturas significan: RAG: relación albúminas / globulinas; AST: aspartato aminotransferasa; CPK: creatin fosfoquinasa; VCM: volumen corpuscular medio; HCM: hemoglobina corpuscular media; CHCM: concentración de hemoglobina corpuscular media.

observar que durante la estación cálida los parámetros analizados fueron más elevados que durante la estación fría. Además, los parámetros hemáticos y pondoestaturales entre machos y hembras dentro de cada especie se comportaron de manera similar, manteniéndose agrupados excepto en C. latirostris durante la estación cálida, donde las hembras mostraron valores morfológicos levemente superiores a los de los machos.

Análisis multivariado de la variancia. Los análisis de la variancia realizados dentro del MANOVA para los efectos especie, sexo e interacción especie por sexo (Tabla 2), reveló que existieron diferencias significativas para el peso y las variables bioquímicas más relevantes (definidas por el ACP anterior) entre las especies C. latirostris y C. yacare (Wilks' Lambda $=0,642, \mathrm{~F}=6,108$; $\mathrm{p}=0,000)$. Sin embargo, no existieron diferencias significativas entre los sexos (Wilks' Lambda $=0,898$, $\mathrm{F}=1,248 ; \mathrm{p}=0,241)$ ni para la interacción entre ambos factores (Wilks' Lambda $=0,938, F=0,720 ; p=0,762$ )
Peso y morfometría. En los ejemplares de C. latirostris, el peso vivo y las dimensiones corporales en la estación cálida resultaron superiores a los de la estación fría. En cambio, en $C$. yacare, el peso vivo, la longitud total, la longitud hocico-cloaca y el perímetro torácico presentaron valores ligeramente más altos en la estación fría que en la cálida, contrariamente a lo observado para la longitud de la cabeza y el ancho de la cabeza (Tabla 3).

A efectos de comparar los datos aquí obtenidos para peso y morfometría, debe acotarse que en base a la longitud total los yacarés se dividen en 4 categorías de importancia biológica: I (0,23-0,40 m: menores de un año), II (0,41-1,30 m: sub-adultos), III (1,31$1,70 \mathrm{~m}$ : reproductores adultos machos y hembras) y IV (> 1,70 m: machos adultos) ${ }^{27}$. Las longitudes totales aquí obtenidas para ejemplares sub-adultos de C. latirostris $(1,04 \mathrm{~m})$ y C. yacare $(1,01 \mathrm{~m})$ no se apartan de dicho marco referencial.

Para ejemplares de $C$. latirostris de 2-4 años de edad mantenidos en un criadero santafesino $(n=160)$, se reportaron longitudes totales $(40-103 \mathrm{~cm})$ cuyo rango mínimo fue sensiblemente inferior al registrado para el conjunto de ambas especies en el presente estudio $(80 \mathrm{~cm})^{21}$. Comparando los valores aquí registrados con los reportados para el cocodrilo australiano de agua salada Crocodylus porosus, surge que el crecimiento de este último es más precoz, pues ejemplares de un año de edad alojados en criaderos $(n=120)$, revelaron rangos máximos de $7,25 \mathrm{~kg}$ de peso y $125 \mathrm{~cm}$ de longitud total ${ }^{14}$.

Al eclosionar del huevo, los neonatos de C. yacare son más pesados que los de C. latirostris (47,7 versus 45,0 g), también poseen mayor longitud total (242 versus $230 \mathrm{~mm}$ ), longitud hocico-cloaca (117 versus 108 $\mathrm{mm}$ ), longitud de cabeza (35 versus $32 \mathrm{~mm}$ ) y ancho de cabeza (20,6 versus 20,2 mm). Sin embargo, en experiencias realizadas en criaderos del nordeste argentino se comprobó que al término de un año, C. latirostris

Tabla 3. Peso y morfometría según estación y especie $(\mathrm{X} \pm \mathrm{DE})$.

\begin{tabular}{lcccc}
\hline \multirow{2}{*}{ Parámetros } & \multicolumn{2}{c}{ C. latirostris } & \multicolumn{2}{c}{ C. yacare } \\
\cline { 2 - 5 } & Estación cálida & Estación fría & Estación cálida & Estación fría \\
\hline Peso vivo $(\mathrm{kg})$ & $4,66 \pm 1,15$ & $4,37 \pm 0,78$ & $3,88 \pm 1,15$ & $3,93 \pm 0,99$ \\
LT $(\mathrm{cm})$ & $104,48 \pm 8,59$ & $102,85 \pm 5,89$ & $101,11 \pm 10,81$ & $101,81 \pm 8,67$ \\
LHC $(\mathrm{cm})$ & $51,07 \pm 4,89$ & $49,73 \pm 3,17$ & $49,21 \pm 4,85$ & $49,39 \pm 4,35$ \\
LC $(\mathrm{cm})$ & $11,83 \pm 0,87$ & $11,46 \pm 0,60$ & $11,89 \pm 1,04$ & $11,73 \pm 0,96$ \\
AC $(\mathrm{cm})$ & $8,05 \pm 0,67$ & $7,75 \pm 0,51$ & $7,59 \pm 0,80$ & $7,26 \pm 0,66$ \\
PT $(\mathrm{cm})$ & $29,77 \pm 3,12$ & $28,62 \pm 2,83$ & $27,27 \pm 3,07$ & $27,33 \pm 2,53$ \\
\hline
\end{tabular}

Las abreviaturas significan: LT: longitud total; LHC: longitud hocico-cloaca; LC: longitud de la cabeza; AC: ancho de la cabeza; PT: perímetro torácico. 
Tabla 4. Proteinograma según estación y especie $(X \pm D E)$.

\begin{tabular}{lcccc}
\hline \multirow{2}{*}{ Parámetros } & \multicolumn{2}{c}{ C. latirostris } & \multicolumn{2}{c}{ C. yacare } \\
\cline { 2 - 5 } & Estación cálida & Estación fría & Estación cálida & Estación fría \\
\hline Proteínas totales $(\mathrm{g} / \mathrm{dl})$ & $4,01 \pm 0,62$ & $3,68 \pm 0,57$ & $4,20 \pm 0,53$ & $3,90 \pm 0,59$ \\
Albúminas $(\mathrm{g} / \mathrm{dl})$ & $0,98 \pm 0,27$ & $1,00 \pm 0,22$ & $0,98 \pm 0,20$ & $0,92 \pm 0,21$ \\
Alfa Globulinas $(\mathrm{g} / \mathrm{dl})$ & $0,77 \pm 0,23$ & $0,74 \pm 0,19$ & $0,73 \pm 0,15$ & $0,73 \pm 0,18$ \\
Beta Globulinas $(\mathrm{g} / \mathrm{dl})$ & $0,85 \pm 0,25$ & $0,78 \pm 0,18$ & $0,92 \pm 0,17$ & $0,97 \pm 0,18$ \\
Gamma Globul. $(\mathrm{g} / \mathrm{dl})$ & $1,42 \pm 0,32$ & $1,18 \pm 0,29$ & $1,55 \pm 0,35$ & $1,29 \pm 0,37$ \\
Globulinas tot. $(\mathrm{g} / \mathrm{dl})$ & $3,03 \pm 0,57$ & $2,70 \pm 0,47$ & $3,20 \pm 0,46$ & $2,99 \pm 0,54$ \\
relación albúm./globul. & $0,32 \pm 0,09$ & $0,37 \pm 0,09$ & $0,31 \pm 0,07$ & $0,31 \pm 0,09$ \\
\hline
\end{tabular}

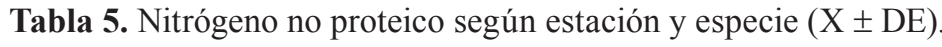

\begin{tabular}{lcccc}
\hline \multirow{2}{*}{ Parámetros } & \multicolumn{2}{c}{ C. latirostris } & \multicolumn{2}{c}{ C. yacare } \\
\cline { 2 - 5 } & Estación cálida & Estación fría & Estación cálida & Estación fría \\
\hline Urea $(\mathrm{mg} / \mathrm{l})$ & $84,9 \pm 33,3$ & $43,0 \pm 29,8$ & $76,7 \pm 34,0$ & $51,7 \pm 29,7$ \\
Creatinina $(\mathrm{mg} / \mathrm{l})$ & $7 \pm 2$ & $5 \pm 2$ & $7 \pm 3$ & $5 \pm 2$ \\
Ácido úrico $(\mathrm{mg} / \mathrm{l})$ & $17 \pm 15$ & $29 \pm 14$ & $20 \pm 13$ & $55 \pm 25$ \\
\hline
\end{tabular}

reveló mayor ganancia de peso (1,44 g/animal/día) que C. yacare ( $0,75 \mathrm{~g} / \mathrm{animal} / \mathrm{dí})$ ), así como mayor aumento de longitud total (667 versus $591 \mathrm{~mm}){ }^{16}$, cambios que ocurrieron en el mismo sentido que el registrado en el presente ensayo.

Pese a que los ejemplares de C. yacare serían más sensibles al frío ${ }^{16}$, en la presente experiencia revelaron pesos mayores en invierno que en verano (diferencia: $+50 \mathrm{~g}$ ), en tanto que en C. latirostris ocurrió lo contrario (diferencia: $-290 \mathrm{~g}$ ). Es ampliamente admitido que durante las épocas de frío ambiental, los caimanes interrumpen o disminuyen la toma de alimentos ("letargo invernal") y restringen su metabolismo hasta una "fase de ahorro" ${ }^{8}$, la cual afecta negativamente la ganancia de peso pues la sub-alimentación de las especies ectotermas provoca el agotamiento de las reservas energéticas (glucógeno, lípidos) y el consumo de proteínas estructurales (músculos), con detención del crecimiento ${ }^{13}$. En el mismo sentido, C. latirostris mantenidos durante dos meses a temperatura ambiental alta $\left(22^{\circ} \mathrm{C}\right)$ revelaron mayores ganancias de peso que los testigos mantenidos a temperatura más baja $\left(18^{\circ} \mathrm{C}\right)^{15}$.

Proteinograma. En C. latirostris, las concentraciones de proteínas totales y de todas las globulinas resultaron más altas en la estación cálida que en la fría. En cambio en C. yacare, las concentraciones de proteínas totales, albúminas, gamma-globulinas y globulinas totales fueron superiores en la estación cálida en comparación a la fría, en la cual los niveles de beta-globulinas alcanzaron los mayores valores. Los valores de alfa-globulinas y la relación albúminas / globulinas no registraron variaciones estacionales (Tabla 4).

En el nordeste argentino otros investigadores obtuvieron valores de proteínas totales y globulinas totales más altos en $C$. latirostris que en $C$. yacare en cautiverio (5,76 versus $5,61 \mathrm{~g} / \mathrm{dl} ; 3,07$ versus $2,30 \mathrm{~g} / \mathrm{dl}$ respectivamente); en cambio, esta última especie registró valores más elevados de albúminas $(2,58$ versus $2,31 \mathrm{~g} /$ dl) ${ }^{24}$. En el presente trabajo los niveles de proteínas totales y albúminas resultaron sensiblemente más bajos, quizás debido a la distinta metodología utilizada para su valoración. Los incrementos de proteínas y globulinas totales aquí registradas en la estación cálida se atribuyen al restablecimiento de la alimentación tras el cese del letargo invernal ${ }^{13}$.

Otros autores reportaron para $C$. latirostris en cautiverio valores más altos de globulinas totales que en ejemplares de vida libre (3,48 versus $3,37 \mathrm{~g} / \mathrm{dl})$, en cambio, en estos últimos registraron niveles más altos de albúminas y de proteínas totales (1,66 versus $1,17 \mathrm{~g} /$ dl y 5,06 versus $4,59 \mathrm{~g} / \mathrm{dl}$ ); por otra parte en C. yacare cautivos observaron concentraciones más altas de albúminas y RAG que en ejemplares silvestres $(0,95 \mathrm{~g} / \mathrm{dl}$ y 0,29 versus $0,87 \mathrm{~g} / \mathrm{dl}$ y 0,23 respectivamente) ${ }^{26}$.

En contraposición a la ausencia de variaciones intersexuales surgentes del presente ensayo, en otras especies (Alligator mississippiensis) se hallaron valores de proteínas totales más bajos en machos $(5,53-5,64$ $\mathrm{g} / \mathrm{dl})$ que en hembras $(5,70-6,20 \mathrm{~g} / \mathrm{dl}){ }^{10}$. En estudios realizados sobre Crocodylus niloticus se reportaron valores más bajos de proteínas totales que los aquí obtenidos para caimanes sudamericanos, a saber: $3,10 \mathrm{~g} /$ dl ${ }^{6}$ y $3,07 \mathrm{~g} / \mathrm{dl}^{20}$.

Nitrógeno no proteico. Tanto en C. latirostris como en C. yacare, las concentraciones de urea y creatinina en sangre resultaron más bajas en la estación fría, mientras que los valores de ácido úrico fueron más altos en la misma época (Tabla 5).

Los mayores niveles registrados en verano para los dos primeros indicadores quizás deban relacionarse al aumento de la ingesta proteica (urea) y al consecuente incremento de las masas musculares (creatinina). Por su parte, los aumentos de ácido úrico en la estación fría podrían atribuirse a la reducción de las funciones hepática y renal como consecuencia del menor tenor metabólico propio del letargo invernal ${ }^{3}$. 
El ácido úrico es el principal residuo del metabolismo nitrogenado de los reptiles; su baja toxicidad permite que sea eliminado en forma de cristales por el riñón ante la escasez de agua, condición ambiental a la cual los caimanes están adaptados ${ }^{4}$. Contradictoriamente a los resultados aquí obtenidos, otros autores comprobaron en ejemplares de criadero que en verano los valores de urea fueron más altos en $C$. yacare que en C. latirostris (122 versus $97,5 \mathrm{mg} / 1$ respectivamente); en cambio, durante la temporada cálida $C$. latirostris superó a C. yacare en los niveles de creatinina y ácido úrico $(3,4 \mathrm{mg} / 1$ y $41,3 \mathrm{mg} / 1$ versus $3,2 \mathrm{mg} / 1$ y $29,4 \mathrm{mg} / 1$, respectivamente). Para ejemplares de vida libre, en la misma publicación se reportaron para ambas especies valores estivales más altos de urea y ácido úrico $(90$ y $137 \mathrm{mg} / \mathrm{l}$, respectivamente) y niveles de creatinina más bajos en ambas especies $(4,5$ y $3,5 \mathrm{mg} / \mathrm{l})$, con relación a los mencionados aquí para la estación cálida ${ }^{26}$.

En ejemplares silvestres de C. latirostris se obtuvieron niveles de nitrógeno no proteico superiores a los de C. yacare (urea: 62,6 versus $51,2 \mathrm{mg} / 1$; creatinina: 3,7 versus $3,5 \mathrm{mg} / 1$; ácido úrico: 35 versus 22 $\mathrm{mg} / \mathrm{l}$, respectivamente) ${ }^{5}$. Hembras de C. latirostris en cautiverio revelaron valores de creatinina de $1,8 \mathrm{mg} / \mathrm{l}$, considerablemente más bajos que los aquí informados ${ }^{25}$. En ejemplares cautivos de A. mississippiensis, especie emparentada con los caimanes aquí estudiados, otros investigadores encontraron valores de ácido úrico de $30 \mathrm{mg} / 1$, más próximos a los aquí obtenidos para $C$. yacare que para C. latirostris ${ }^{1}$.

Glucosa y lipidograma. En ambas especies estudiadas, los niveles sanguíneos de glucosa, colesterol total, triglicéridos, C-HDL y lipoproteínas beta fueron superiores en la estación cálida, mientras que C-LDL y lipoproteínas alfa, lo fueron en la época fría (Tabla 6).

Los niveles de glucosa y triglicéridos de los caimanes, como era predecible debido a la disminución de la ingesta de alimentos ${ }^{3}$, resultaron más bajos en la estación fría que en la cálida, en ambas especies. Este comportamiento parecería estar sometido a un ritmo circanual pues ocurre incluso en animales sometidos a temperatura ambiental controlada ${ }^{9}$, como es el caso de los ejemplares aquí estudiados.

Inversamente a lo ocurrido en el presente trabajo, otros autores encontraron en C. latirostris en cautiverio concentraciones de colesterol total más altas en la estación fría que en la cálida ( 3,0 versus $1,5 \mathrm{~g} / \mathrm{l})$, aunque los niveles de C-HDL fueron más altos en la estación cálida $(0,61$ versus $0,36 \mathrm{~g} / \mathrm{l})$ y los de C-LDL se encontraron más elevados en la estación fría (1,76 versus $0,60 \mathrm{~g} / \mathrm{l})$, en concordancia con lo registrado en el presente trabajo ${ }^{21}$. En coincidencia con nuestros resultados, en otro ensayo realizado con reptiles en cautiverio se reportaron valores más altos de glucosa en C. latirostris que en C. yacare $(0,98$ versus $0,77 \mathrm{~g} / \mathrm{l})$ y valores más altos de colesterol total en C. yacare que en C. latirostris $(0,79$ versus $0,68 \mathrm{~g} / 1)$; en contraposición, en dicho ensayo se hallaron niveles de triglicéridos más bajos en $C$. yacare que en $C$. latirostris $(1,45 \text { versus } 1,98 \mathrm{~g} / 1)^{24}$.

En A. mississippiensis fueron encontrados valores de glucosa cercanos a los hallados aquí para C. yacare en temporada cálida $(0,73 \mathrm{~g} / \mathrm{l}){ }^{19}$. En la misma especie otro investigador comunicó la semejanza entre las glucemias de machos y hembras silvestres $(1,23$ y $1,21 \mathrm{~g} / 1$ respectivamente), que resultaron marcadamente supe-

Tabla 6. Glucosa y Lipidograma según estación y especie (X $\pm \mathrm{DE})$.

\begin{tabular}{lcccc}
\hline \multirow{2}{*}{ Parámetros } & \multicolumn{2}{c}{ C. latirostris } & \multicolumn{2}{c}{ C. yacare } \\
\cline { 2 - 5 } & Estación cálida & Estación fría & Estación cálida & Estación fría \\
\hline Glucosa $(\mathrm{g} / \mathrm{l})$ & $0,91 \pm 0,27$ & $0,52 \pm 0,10$ & $0,79 \pm 0,24$ & $0,50 \pm 0,17$ \\
Colesterol total $(\mathrm{g} / \mathrm{l})$ & $0,34 \pm 0,17$ & $0,24 \pm 0,13$ & $0,55 \pm 0,19$ & $0,31 \pm 0,13$ \\
Triglicéridos $(\mathrm{g} / \mathrm{l})$ & $0,66 \pm 0,48$ & $0,30 \pm 0,21$ & $0,81 \pm 0,51$ & $0,33 \pm 0,22$ \\
C-HDL $(\mathrm{g} / \mathrm{l})$ & $0,03 \pm 0,03$ & $0,02 \pm 0,01$ & $0,06 \pm 0,05$ & $0,02 \pm 0,01$ \\
C-LDL $(\mathrm{g} / \mathrm{l})$ & $0,10 \pm 0,08$ & $0,16 \pm 0,09$ & $0,17 \pm 0,12$ & $0,18 \pm 0,09$ \\
Lipo alfa (\%) & $82 \pm 6$ & $88 \pm 5$ & $82 \pm 5$ & $85 \pm 7$ \\
Lipo beta (\%) & $18 \pm 6$ & $12 \pm 5$ & $18 \pm 5$ & $15 \pm 7$ \\
\hline
\end{tabular}

Las abreviaturas significan: C-HDL: colesterol ligado a lipoproteínas de alta densidad, C-LDL: colesterol ligado a lipoproteínas de baja densidad, Lipo: lipoproteína.

Tabla 7. Ionograma según estación y especie $(\mathrm{X} \pm \mathrm{DE})$.

\begin{tabular}{lcccc}
\hline \multirow{2}{*}{ Parámetros } & \multicolumn{2}{c}{ C. latirostris } & \multicolumn{2}{c}{ C. yacare } \\
\cline { 2 - 5 } & Estación cálida & Estación fría & Estación cálida & Estación fría \\
\hline Sodio $(\mathrm{mEq} / \mathrm{l})$ & $150 \pm 6$ & $149 \pm 7$ & $152 \pm 4$ & $147 \pm 9$ \\
Potasio $(\mathrm{mEq} / \mathrm{l})$ & $5,14 \pm 0,56$ & $4,36 \pm 0,74$ & $4,88 \pm 0,58$ & $3,97 \pm 0,63$ \\
Calcio $(\mathrm{mg} / \mathrm{dl})$ & $9,00 \pm 1,07$ & $8,68 \pm 0,98$ & $8,92 \pm 0,94$ & $8,32 \pm 1,32$ \\
Fósforo $(\mathrm{mg} / \mathrm{dl})$ & $4,54 \pm 1,52$ & $3,72 \pm 0,89$ & $4,86 \pm 1,26$ & $3,73 \pm 0,96$ \\
Magnesio $(\mathrm{mg} / \mathrm{dl})$ & $2,70 \pm 0,28$ & $2,76 \pm 0,25$ & $2,56 \pm 0,33$ & $2,45 \pm 0,31$ \\
Cobre $(\mathrm{ug} / \mathrm{dl})$ & $84 \pm 36$ & $131 \pm 33$ & $83 \pm 36$ & $156 \pm 25$ \\
\hline
\end{tabular}


Tabla 8. Enzimograma según estación y especie (X $\pm \mathrm{DE})$.

\begin{tabular}{lcccc}
\hline \multirow{2}{*}{ Parámetros } & \multicolumn{2}{c}{ C. latirostris } & \multicolumn{2}{c}{ C. yacare } \\
\cline { 2 - 5 } & Estación cálida & Estación fría & Estación cálida & Estación fría \\
\hline ALP (UI/l) & $48 \pm 26$ & $56 \pm 19$ & $50 \pm 24$ & $57 \pm 25$ \\
ALT (UI/l) & $13 \pm 6$ & $14 \pm 5$ & $14 \pm 7$ & $12 \pm 7$ \\
AST (UI/l) & $53 \pm 15$ & $66 \pm 20$ & $82 \pm 35$ & $110 \pm 47$ \\
GGT (UI/l) & $10 \pm 6$ & $11 \pm 5$ & $9 \pm 5$ & $7 \pm 6$ \\
CPK (UI/l) & $113 \pm 74$ & $152 \pm 116$ & $184 \pm 104$ & $255 \pm 134$ \\
LDH (UI/l) & $356 \pm 150$ & $277 \pm 156$ & $425 \pm 174$ & $386 \pm 177$ \\
CHE (UI/l) & $555 \pm 179$ & $307 \pm 163$ & $653 \pm 223$ & $347 \pm 151$ \\
\hline
\end{tabular}

Las abreviaturas significan: ALP: fosfatasa alcalina; ALT: alanin aminotrasferasa; AST: aspartato aminotransferasa; GGT: gammaglutamil transferasa; CPK: creatin fosfoquinasa; LDH: lactato deshidrogenasa; CHE: butiril colinesterasa.

riores a las halladas en ejemplares en cautiverio $(0,73 \mathrm{y}$ $0,77 \mathrm{~g} / 1$ respectivamente), estas últimas más cercanas a las reportadas en este ensayo ${ }^{12}$.

Ionograma. Para C. latirostris, sodio, potasio, calcio y fósforo resultaron con valores más altos en la estación cálida, en tanto que magnesio y cobre fueron mayores en la estación fría. Por otra parte, en C. yaca$r e$ sólo este último electrolito presentó concentraciones invernales más altas (Tabla 7).

Durante la presente experiencia, la más alta concentración sérica de la mayoría de los electrolitos en el período primavera-verano quizás responda al incremento de la ingestión de alimentos, como está descripto en otras especies ${ }^{13}$. En el nordeste argentino, otros estudios demostraron que durante el verano, ejemplares cautivos de $C$. latirostris y $C$. yacare ostentaron valores semejantes de sodio ( 150 y $148 \mathrm{meq} / 1$ respectivamente) y potasio $(4,43$ y $4,58 \mathrm{meq} / \mathrm{l})$, pero considerablemente más altos de calcio $(13,51$ y $10,54 \mathrm{mg} / \mathrm{dl})$ y fósforo inorgánico (7,78 y $5,16 \mathrm{mg} / \mathrm{dl}$ respectivamente) ${ }^{26}$. Tales diferencias podrían atribuirse al tipo de alimento o distinta técnica de laboratorio para la determinación ${ }^{3}$.

En otros trabajos se reportan valores más altos de calcio y fósforo inorgánico en $C$. latirostris que en $C$. yacare ( 9,63 versus $7,03 \mathrm{mg} / \mathrm{dl}$ y 5,08 versus $4,27 \mathrm{mg} / \mathrm{dl}$ respectivamente); esta última especie presentó valores más altos de sodio y potasio que la primera (114 versus 109 meq/l y 4,38 versus 4,02 meq/1 respectivamente) ${ }^{24}$. En ejemplares en cautiverio de C. latirostris se hallaron niveles más altos de sodio, potasio, calcio y fósforo inorgánico que en $C$. yacare (144,06 meq/1; 4,6 meq/l; $8,9 \mathrm{mg} / \mathrm{dl}$ y $5,64 \mathrm{mg} / \mathrm{dl}$ versus $129,33 \mathrm{meq} / \mathrm{l} ; 3,51 \mathrm{meq} / \mathrm{l}$; $5,86 \mathrm{mg} / \mathrm{dl}$ y $3,58 \mathrm{mg} / \mathrm{dl}$ respectivamente) ${ }^{5}$. En cambio, en el presente ensayo se obtuvieron concentraciones de potasio, calcio y magnesio más altas en $C$. latirostris que en C. yacare.

En ejemplares silvestres de A. mississippiensis de sexo macho se reportaron niveles más altos de calcio y magnesio $(12,48$ y $3,21 \mathrm{mg} / \mathrm{dl}$ respectivamente) y más bajos de cobre (76 ug/dl) que los obtenidos aquí para los caimanes del nordeste argentino ${ }^{10}$. Los valores consignados ut supra resultaron superiores a los encontrados en ejemplares cautivos de la misma especie: calcio y magnesio: 11,48 y $2,58 \mathrm{mg} / \mathrm{dl}$ respectivamente y cobre: $60 \mathrm{ug} / \mathrm{dl}{ }^{11}$.

Enzimograma. C. latirostris presentó actividades séricas de ALP, ALT, AST, GGT y CPK más altas en la estación fría; en cambio, LDH y CHE fueron superiores en la estación cálida. Por su parte, C. yacare mostró concentraciones más altas ALP, AST y CPK en la época fría y el resto de las enzimas fueron superiores en temporada estival (Tabla 8).

En líneas generales los cambios de las actividades enzimáticas en el suero de los reptiles deben ser interpretadas de la misma manera que para mamíferos y aves ${ }^{2}$. El aumento de ALP usualmente indica una mayor actividad osteoblástica pero también puede ocurrir por colestasis. Las elevaciones de ALT y AST indicarían daño hepático o muscular. La enzima GGT puede originarse en tejidos hepático, pancreático y renal, por lo cual su variación plasmática estaría relacionada con trastornos en estos órganos. CPK proviene del tejido nervioso y de los músculos esquelético y cardíaco. LDH es de origen hepático y también muscular; su aumento estaría indicando destrucciones tisulares. CHE se origina en el hígado y su disminución en el plasma indica insuficiencia de este órgano o intoxicación por productos organofosforados ${ }^{3}$.

Comparados con C. yacare, ejemplares cautivos de C. latirostris registraron niveles más altos de ALT (18 versus $14 \mathrm{UI} / \mathrm{l})$, AST (135 versus $92 \mathrm{UI} / \mathrm{l})$ y GGT (16 versus $15 \mathrm{UI} / 1)$; en cambio C. yacare reveló mayores actividades séricas de ALP (105 versus $30 \mathrm{UI} / \mathrm{l}$ ) y LDH (1911 versus $1348 \mathrm{UI} / 1)^{24}$. Con relación a las actividades enzimáticas obtenidas en el presente ensayo, otros autores informaron para C. latirostris y C. yacare en temporada de verano, actividades más bajas de ALP (24 y 18 UI/1 respectivamente) y de LDH (338 y 135 UI/l) y más altas de ALT (57 y 20 UI/l), AST (118 y 49 UI/l) y CPK (375 y 340 UI/l, respectivamente) ${ }^{26}$. Comparando los valores aquí obtenidos para ambas especies de caimanes, surge que los niveles de CPK, LDH y CHE fueron más bajos que los reportados para hembras cautivas de C. latirostris (406; 3128 y 1056 $\mathrm{UI} / 1$ respectivamente) ${ }^{25}$. 
Eritrograma. En ambas especies aquí estudiadas, los parámetros que conforman el eritrograma mostraron valores más altos en la estación cálida que en la fría, con excepción de la concentración de glóbulos rojos y la CHCM, parámetros que no presentaron variaciones estacionales en C. yacare (Tabla 9).

Las fluctuaciones estacionales de la serie roja registradas en esta experiencia deben atribuirse a la optimización del estado nutricional propio de la estación cálida y a su detrimento durante el período frío. Así, se ha reportado que aunque a estos reptiles se les suministre la misma cantidad de alimento durante todo el año, la baja temperatura ambiental interfiere en el proceso digestivo debido a una menor secreción de enzimas en el canal alimentario ${ }^{22}$.

En otra investigación sobre C. latirostris en cautiverio se obtuvieron valores disímiles a los aquí registrados. Así, los valores de verano e invierno fueron: hematocrito: 24 versus $22 \%$, VCM: 455 versus $373 \mathrm{fl}$, hemoglobina: 11,6 versus 8,6 g/dl, HCM: 201 versus $167 \mathrm{pg}$ y CHCM: 53 versus $37 \%{ }^{23}$. Con relación a los valores registrados en la presente investigación para ambas especies en conjunto, otros investigadores hallaron en verano niveles más altos de hematocrito para C. latirostris y C. yacare, tanto en cautiverio $(24,8$ y $22,17 \%$ respectivamente) como silvestres (24 y $23 \%)^{26}$.

En el nordeste argentino se indagaron los valores del hematocrito de ejemplares en cautiverio de ambas especies autóctonas, resultando más altos en C. latirostris que en C. yacare (21,94 versus $18,41 \%)$; en cambio en ejemplares silvestres ocurrió a la inversa pues $C . y a-$ care registró valores más altos que C. latirostris $(29,93$ versus $22,97 \%)^{5}$. En otra especie americana, Caiman crocodilus, se reportaron rangos más altos de hematocrito y eritrocitos $(20-30 \%$ y $0,50-0,64 \mathrm{~T} / 1$ respectiva- mente) y valores más bajos de índices hematimétricos (VCM: 42,64 fl; HCM: 11,39 pg; CHCM: 26,85\%) que los obtenidos en el presente ensayo ${ }^{17}$. Nuevamente parece haberse deslizado un error al escribir algunas de las cifras precedentes; interpretamos que el VCM debe ser $426 \mathrm{fl} \mathrm{y} \mathrm{la} \mathrm{HCM} 113 \mathrm{pg}$.

Leucograma. En lo atinente a los glóbulos blancos, en ambas especies aquí estudiadas el recuento total de leucocitos y los porcentajes de heterófilos y monocitos resultaron más bajos en la estación cálida, durante la cual fueron más altos los porcentajes de linfocitos, sin que se registraran variaciones de eosinófilos ni basófilos (Tabla 10).

Se asevera que las variaciones estacionales de los glóbulos blancos estarían relacionadas a los cambios de temperatura ambiental, oscilaciones del fotoperíodo y conducta alimentaria. Así, en ejemplares juveniles de C. latirostris de ambos sexos, se hallaron diferencias leucocitarias entre invierno y otoño; los machos presentaron valores más altos de leucocitos totales en invierno (10,00 versus $5,17 \mathrm{G} / 1)$ y las hembras en otoño (9,63 versus 14,50 G/l). Asimismo, tanto en otoño como en invierno los machos adultos registraron valores más altos de leucocitos totales $(9,77$ y $9,47 \mathrm{G} / 1$, respectivamente) que las hembras adultas $(9,67 \text { y } 8,61 \mathrm{G} / 1)^{7}$.

En ejemplares de $C$. latirostris se encontraron valores más altos en invierno que en verano para leucocitos totales y heterófilos $(24,36$ versus 22,68 G/l y 5 versus $2 \%$, respectivamente), en cambio en la estación cálida los porcentajes de linfocitos fueron más altos que en invierno (68 versus 66\%), en tanto que eosinófilos, monocitos y basófilos no registraron diferencias estacionales (19; 5 y $1 \%$ respectivamente) ${ }^{23}$. En ejemplares silvestres de C. crocodilus fueron hallados valores más bajos

Tabla 9. Eritrograma según estación y especie $(X \pm D E)$.

\begin{tabular}{lcccc}
\hline \multirow{2}{*}{ Parámetros } & \multicolumn{2}{c}{ C. latirostris } & \multicolumn{2}{c}{ C. yacare } \\
\cline { 2 - 5 } & Estación cálida & Estación fría & Estación cálida & Estación fría \\
\hline Hematocrito (\%) & $21 \pm 4$ & $19 \pm 3$ & $21 \pm 3$ & $20 \pm 4$ \\
Eritrocitos (T/l) & $0,47 \pm 0,10$ & $0,46 \pm 0,10$ & $0,51 \pm 0,10$ & $0,51 \pm 0,10$ \\
VCM (fl) & $442 \pm 44$ & $425 \pm 42$ & $429 \pm 44$ & $405 \pm 36$ \\
Hemoglobina (g/dl) & $6,22 \pm 1,42$ & $5,58 \pm 1,28$ & $5,87 \pm 1,48$ & $5,52 \pm 1,39$ \\
HCM (pg) & $133 \pm 23$ & $122 \pm 16$ & $116 \pm 22$ & $109 \pm 18$ \\
CHCM (\%) & $30 \pm 5$ & $28 \pm 4$ & $27 \pm 5$ & $27 \pm 3$ \\
\hline
\end{tabular}

Las abreviaturas significan: VCM: volumen corpuscular medio; HCM: hemoglobina corpuscular media; CHCM: concentración de hemoglobina corpuscular media.

Tabla 10. Leucograma según estación y especie $(X \pm D E)$.

\begin{tabular}{lcccc}
\hline \multirow{2}{*}{ Parámetros } & \multicolumn{2}{c}{ C. latirostris } & \multicolumn{2}{c}{ C. yacare } \\
\cline { 2 - 5 } & Estación cálida & Estación fría & Estación cálida & Estación fría \\
\hline Leucocitos (G/l) & $13,24 \pm 3,80$ & $14,02 \pm 4,69$ & $13,47 \pm 3,38$ & $14,81 \pm 5,59$ \\
Linfocitos (\%) & $83 \pm 7$ & $65 \pm 11$ & $76 \pm 9$ & $68 \pm 11$ \\
Eosinófilos (\%) & $2 \pm 1$ & $2 \pm 1$ & $2 \pm 1$ & $2 \pm 1$ \\
Heterófilos (\%) & $11 \pm 6$ & $25 \pm 10$ & $18 \pm 8$ & $23 \pm 11$ \\
Monocitos (\%) & $4 \pm 2$ & $6 \pm 4$ & $4 \pm 2$ & $7 \pm 3$ \\
Basófilos (\%) & $1 \pm 1$ & $1 \pm 1$ & $0 \pm 0$ & $0 \pm 0$ \\
\hline
\end{tabular}


de leucocitos totales y linfocitos $(10,40 \mathrm{G} / 1$ y $20 \%$ respectivamente) y tasas más altas de monocitos $(7,84 \%)$ y granulocitos (eosinófilos: 15,30\%; heterófilos: 51,36\%; basófilos: $4,80 \%$ ) con relación a los registrados en el presente trabajo ${ }^{17}$.

En ejemplares cautivos de A. mississippiensis se hallaron valores más bajos de leucocitos totales y linfocitos $(5,80 \mathrm{G} / 1$ y $11,1 \%$, respectivamente), así como porcentajes más altos de eosinófilos $(9,2 \%)$, heterófilos $(57,6 \%)$, basófilos $(1,2 \%)$ y monocitos $(5,2 \%)$, que los obtenidos en la presente investigación ${ }^{1}$. En cambio, en ejemplares silvestres de la misma especie otros autores encontraron niveles inferiores de leucocitos $(12,26 \mathrm{G} / \mathrm{l})$ y linfocitos (33\%); valores superiores de eosinófilos $(12 \%)$, monocitos $(12 \%)$ y basófilos $(8 \%)$. Para los heterófilos (considerados como "neutrófilos") se registró una tasa de $35 \%$, la cual supera el guarismo obtenido en este trabajo ${ }^{18}$.

En conclusión, se obtienen valores de referencia para la etapa juvenil (categoría sub-adultos) de ambas especies de caimanes autóctonos del nordeste argentino mantenidos en cautiverio y alimentados con la misma dieta. Si bien no se registraron diferencias significativas entre sexos, debe tenerse en cuenta que a esa edad aún no se manifiesta el comportamiento reproductivo. El peso y las dimensiones morfométricas variaron según la especie y época del año. Las estadísticas multivariadas detectaron diferencias fisiológicas entre las concentraciones de los componentes sanguíneos de $C$. latirostris y C. yacare, así como cambios atribuibles a la temperatura ambiental. El hecho que la mayoría de los parámetros metabólico-nutricionales resultara superior en la estación cálida, permite presumir un cambio en la magnitud de la actividad metabólica de estos animales ectodermos, que si bien permanecieron alojados en recintos protegidos de la intemperie y con calefacción en las épocas frías, no estuvieron exentos de la influencia del fotoperíodo ni del "reloj biológico filogenético" identificador de las estaciones del año. Se espera que tales conocimientos puedan ser aplicados para optimizar el sistema de cría de caimanes autóctonos.

\section{REFERENCIAS}

1. Barnett JD, Cardeilhac PT, Barr B, Wolf W, Bass OL, Fleming DM. 1998. Utilization of thyroid hormone levels to determinate starvation in alligators from the Everglades National Park. Proceed Internat Ass Aquatic Anim Med 29: 52-56.

2. Campbell TW. 1996. Clinical pathology. In: Reptile medicine and surgery (Mader DR Ed.), Saunders, Philadelphia, p. 248-257.

3. Coppo JA. 2008. Fisiología comparada del medio interno, EUCASA, Salta (Argentina), 309 p.

4. Eckert R, Randall D, Augustine G. 1992. Fisiología animal, McGraw-Hill, Madrid, 795 p.

5. Ferreyra H, Uhart M. 2001. Evaluación y evolución del estado sanitario de Caiman latirostris y Caiman yacare en el Refugio El Cachapé. Boletín Técnico de la Fundación Vida Silvestre Argentina 55: 1-15.

6. Foggin CM. 1987. Diseases and disease control on crocodile farms in Zimbabwe. In: Wildlife management: crocodiles and alligators (Webb GJ, Manolis SC, Whitehead PJ, Ed.), Surrey Beatty \& Sons, Chipping Norton (UK), p. 351-362.

7. García PB, Matushima ER, Ramos MC, Dias JL, Verdade LM. 1993. Variações sazonais do padrão hematológico e protéico de jacarés de papo amarelo (Caiman latirostris) em cativeiro. Anais do III Workshop sobre Conservação e Manejo do jacaré do papo amarelo, Piracicaba (São Paulo, Brasil), p. 51-60.

8. Hoar WS. 1983. General and comparative physiology, 3rd ed., Prentice-Hall, New Jersey, 848 p.

9. Huchzermeyer FW. 2003. Crocodiles: biology, husbandry and diseases, CAB International, London, $337 \mathrm{p}$.

10. Lance VA, Joanen T, McNease L. 1983. Selenium, vitamin E, and trace elements in the plasma of wild and farmreared alligators during the reproductive cycle. Canadian J Zool 61: 1744-1751.

11. Lance VA, Lauren D. 1984. Circadian variation in plasma corticosterone in the american alligator, Alligator mississippiensis, and the effects of ACTH injections. Gen Comp Endocrinol 54: 1-7.

12. Lance VA, Morici LA, Elsey RM, Lund ED, Place AR. 2001. Hyperlipidemia and reproductive failure in captive-reared alligators: vitamin E, vitamin A, plasma lipids, fatty acids, and steroid hormones. Comp Biochem Physiol B Biochem Mol Biol 128: 285-294.

13. Machado CR, Garófalo MA, Roselino JE, Kettelhut IC, Migliorini RH. 1988. Effects of starvation, refeeding, and insulin on energy-linked metabolic processes in catfish (Rhamdia hilarii) adapted to a carbohydrate-rich diet. Gen Comp Endocrinol 71: 429-437.

14. Millan JM, Janmaat A, Richardson KC, Chambers LK, Fomiatti KR. 1997. Reference ranges for biochemical and haematological values in farmed saltwater crocodile (Crocodylus porosus) yearlings. Aust Vet J 75: 814-817.

15. Piña C, Larriera A. 2002. Caiman latirostris growth: the effect of a management technique on the supplied temperature. Aquaculture 211: 387-392.

16. Prado W, Gómez O, Balla P. 2001. Crianza en cautiverio y reintroducción de individuos. Boletín Técnico de la Fundación Vida Silvestre Argentina 55: 26-38.

17. Rossini VM. 2004. Determinación de los parámetros hematológicos de la baba (Caiman crocodilus) en hábitat silvestre. Anales XIX Panamerican Congress of Veterinary Sciences, Buenos Aires, p. 214.

18. Schoeb TR, Heaton-Jones TG, Clemmons RM, Carbonneau DA, Woodward AR, Shelton D, Poppenga RH. 2002. Clinical and necropsy findings associated with increased mortality among american alligators of lake Griffin, Florida. J Wildlife Dis 38: 320-337.

19. Stein G. 1996. Hematologic and blood chemistry values in reptiles. In: Reptile medicine and surgery (Mader DR Ed.), Saunders, Philadelphia, p. 118-152.

20. Swanepoel D, Boomker J, Kriek NP. 2000. Selected chemical parameters in the blood and metals in the organs 
of the Nile crocodile, Crocodylus niloticus, in the Kruger National Park. Onderstepoort J Vet Res 67: 141-148.

21. Tourn S, Imhof A, Costa A, von Finck C, Barriera A. 1993. Colecta de sangre y procesamiento de muestras en Caiman latirostris. Memorias IV Workshop sobre Conservación y Manejo de Yacaré Overo, Caiman latirostris, Santo Tomé (Santa Fe, Argentina), p. 25-30.

22. Troiano JC. 1991. Manejo sanitario de reptiles en cautiverio, Prensa Veterinaria Argentina, Buenos Aires, 176 p.

23. Troiano JC, Althaus R. 1993. Hallazgos hematológicos en Caiman latirostris (Crocodylia-Alligatoridae) en condiciones de cautiverio. Memorias IV Workshop sobre Conservación y Manejo de Yacaré Overo, Caiman latirostris, Santo Tomé (Santa Fe, Argentina), p. 12-24.

24. Troiano JC, Althaus RL, Malinskas G. 1997. Perfil bioquímico sanguíneo de las especies del género Caiman en condiciones de cautividad. Rev Españ Herpetol 11: 31-34.
25. Trossero SM, Siroski P, Piña CI. 2005. Variación estacional del perfil bioquímico en hembras juveniles de Caiman latirostris criadas en cautiverio. Proceedings Reunión Regional de América Latina y el Caribe del CSG/ SSC/IUCN (Santa Fe, Argentina), p. 220-233.

26. Uhart M, Prado W, Beldoménico P, Rossetti C, Ferryera MC, Martinez A, Bardón JC, Avilés G, Karesh W. 2001. Estudios sanitarios comparativos de yacarés (Caiman latirostris y Caiman yacare) silvestres y cautivos. Boletín Técnico Fundación Vida Silvestre Argentina 55: 1-15.

27. Waller T, Minucci PA. 1993. Relevamiento de la distribución, hábitat y abundancia de los crocodilios de la Provincia de Corrientes, Argentina. Memorias 1ra. Reunión Regional del Grupo de Especialistas en Cocodrilos, Santa Marta (Colombia), p. 341-385.

\section{Asociación Cooperadora de la Facultad de Ciencias Veterinarias}

Personería Jurídica No 647/92 y 912/00

Sargento Cabral 2139

3400 Corrientes

La Asociación Cooperadora de la Facultad de Ciencias Veterinarias fue constituida el 10 de diciembre del año 1991 como entidad de bien público, con el objeto de promover y coadyuvar las actividades científicas, educativas y culturales relacionadas con las Ciencias Veterinarias. En tal sentido, implementa acciones para colaborar con la enseñanza, extensión, actualización y difusión científica que realiza dicha Casa de Estudios.

Beneficios que brinda a sus asociados:

- Fotocopias con descuentos especiales del 20\% en la Fotocopiadora COPIAS.COM que funciona dentro del predio de la Facultad de Ciencias Veterinarias.

- $10 \%$ de descuento para la adquisición de libros de la Editorial Inter-Médica.

- $10 \%$ de descuento en las compras de medicamentos e insumos para trabajos prácticos hospitalarios. 


\title{
Combinación analgésica utilizada en perras sometidas a ovariohisterectomía
}

\author{
López, J.E.; Guaimás Moya, L.E.; Báez, A.D.; Lockett, M.B.; Repetto, C.J. \\ Hospital de Clínicas y Cátedra de Cirugía y Anestesiología, Facultad de Ciencias Veterinarias, Universidad \\ Nacional del Nordeste (UNNE), Sargento Cabral 2139, Corrientes (3400), Argentina. Tel/fax 03783-425753. \\ E-mail: tecquir@vet.unne.edu.ar.
}

\begin{abstract}
Resumen
López, J.E.; Guaimás Moya, L.E.; Báez, A.D.; Lockett, M.B.; Repetto, C.J.: Combinación analgésica utilizada en perras sometidas a ovariohisterectomía. Rev. vet. 21: 2, 123-126, 2010. La analgesia perioperatoria es uno de los requisitos fundamentales del éxito de una cirugía, conjuntamente a una técnica quirúrgica adecuada. El manejo correcto del dolor post-operatorio comienza en la etapa pre-operatoria, durante la cual las drogas sistémicas pueden ser administradas en forma programada y repetirlas las veces que sea necesario. El dolor ha sido definido como una experiencia sensoria y emocional desagradable, asociada con daño tisular real o potencial. Debido a inconvenientes en la medición del dolor animal, las instituciones pautaron que si un procedimiento quirúrgico ocasiona dolor en pacientes humanos adultos, los animales de laboratorio sometidos al mismo deben ser tratados con terapia analgésica. El objetivo del trabajo fue evaluar los efectos de una combinación analgésica conformada por dextropropoxifeno, lidocaina, xilacina y ketamina en 15 perras sometidas a ovariohisterectomía. La premedicación fue realizada con dextropropoxifeno y xilacina, ambas por vía subcutánea, la primer droga se administró treinta minutos antes que la segunda, mientras que la inducción se llevó a cabo con propofol y diazepam. La perfusión de la combinación ensayada comenzó luego de la intubación endotraqueal, mediante la cual un vaporizador de isoflurano suministró una concentración inicial de 3\% la cual fue variando según los requerimientos del animal (1,5-3\%). El oxígeno se aportó a razón de $20 \mathrm{ml} / \mathrm{kg}$ / min. No se registraron modificaciones importantes de parámetros como temperatura rectal y electrocardiografía, observándose algunas modificaciones en frecuencia cardiaca, respiratoria y presión arterial media (directa), las cuales fueron evaluadas en 8 tiempos distintos que se iniciaron 5 minutos antes de la premedicación anestésica. Hacia la mitad de la experiencia, la frecuencia respiratoria se registró en los límites inferiores. La presión arterial acusó elevaciones iniciales coincidentes con los momentos de mayor estimulación nociceptiva. Se concluye que la combinación ensayada produjo una adecuada analgesia sin causar alteraciones destacables de la estabilidad cardiorrespiratoria.
\end{abstract}

Palabras clave: perra, ovariohisterectomía, analgesia, dextropropoxifeno, lidocaina, xilacina, ketamina.

\begin{abstract}
López, J.E.; Guaimás Moya, L.E.; Báez, A.D.; Lockett, M.B.; Repetto, C.J.: Analgesic combination for ovariohysterectomy in the bitch. Rev. vet. 21: 2, 123-126, 2010. The perioperative analgesia must be considered as a fundamental requirement for a successful surgery, together with the appropriate surgery technique. The correct way for controlling the postoperative pain starts in the preoperative stage where systemic drugs can be administrated in a programmed way and can be repeated if necessary. Pain is a sensorial and emotional unpleasant experience, associated with real or potential tisular damage. Due to inconvenients in measuring animal pain, medical institutions established that if a surgical procedure causes pain in adult human patients, laboratory animals submitted to the same procedure must be treated with analgesic therapy. The objective of this trial was to evaluate the effects of an analgesic combination with dextropropoxiphene, lidocaine, xylazine and ketamine on 15 female dogs submitted to ovariohysterectomy. Pre-medication was made with dextropropoxiphene and xylazine both by subcutaneous way. Dextropropoxiphene was administrated thirty minutes before xylazine, and then induction was made with propofol and diazepam. The perfusion of the drug combination started after the endotracheal intuba-
\end{abstract}

